\title{
Nuclease-based gene drives, an innovative tool for insect vector control: advantages and challenges of the technology
}

\author{
Charlotte M. Quinn and Tony Nolan* \\ Liverpool School of Tropical Medicine \\ *corresponding author: tony.nolan@Istmed.ac.uk
}

\begin{abstract}
Genetic control of insects involves the release of modified insects that contain altered genetic traits and are competent to mate with target populations to introduce the traits therein. Since it relies on mating, this type of control is species-specific, non-toxic, and has the advantage that the released insects can do the difficult task of reaching remote and otherwise inaccessible insect niches.
\end{abstract}

Gene drives are capable of drastically biasing their own transmission and are being developed as a new type of genetic control, one that would be self-sustaining, requiring low numbers in the initial release in order to spread and persist within a population. In this review, the advantages and challenges of building and deploying this technology will be discussed, using mosquito control as an example.

\section{Control of Insect Vectors of Disease}

Several severe diseases of humans are transmitted only by the bites of insects as they seek a blood source for nutrition and the nurturing of eggs in order to complete their own life cycle. Collectively, these insect 'vectors' are responsible for over 700,000 human deaths every year through the parasitic or viral diseases that they transmit (World Health Organisation 2017), including malaria, dengue, lymphatic filariasis, Chagas disease, onchocerciasis, leishmaniasis, chikungunya, Zika, yellow fever, and Japanese encephalitis. For the most severe of these diseases, such as Dengue and malaria transmitted by Aedes and Anopheles mosquitoes respectively, highly effective vaccines do not exist and success stories of lowering the disease burden have predominantly been due to vector control (Bhatt et al. 2015; World Health Organisation 2019). Elimination of any disease is likely to require a combined approach of reducing the transmission potential of vector populations at the same time as removing parasite reservoirs in the human population. However, the proven gains and reduction in disease burden as a direct result of vector control mean that a huge amount of research and investment has focused on a range of strategies to reduce mosquito populations.

For any vector control program, high quality entomological data are an essential starting point since this will affect the suitability and feasibility of different interventions. Key questions include: When is biting (and thus transmission) occurring? Where is biting occurring (inside or outside the home)? How many vector species are responsible for transmission in a given area? Where are the larval breeding sites? What is the geographical distribution of vector populations (urban or rural)? What is the insecticide susceptibility of target populations? What fraction of vectors are diseasecarriers?

In this review we will use the control of Anopheles mosquitoes as a paradigm for the relevance of some of the above questions and the prospects of novel genetic control approaches such as gene drive, in context with conventional vector control, though the general principles are applicable to a wide range of insect vectors.

\section{Genetic control versus 'conventional' vector control strategies}


Vector control strategies for malaria control have included interventions such as indoor residual spraying (IRS), insecticide-treated barriers e.g. bed nets and screens, habitat and breeding source removal, and larviciding; they are generally inexpensive but highly effective, low-tech methods of insect population control and have so far played a paramount role in the prevention and elimination of many vector-borne diseases. The lion's share of success has been attributed particularly to the use of insecticide-treated bednets (ITNs) and IRS (Bhatt et al. 2015). However, several limitations are quickly becoming apparent: screens and bed nets do not protect against outdoor biting, with the efficacy of the latter mitigated more so by only providing protection at night; the lack of specificity and toxicity of insecticides and fumigatory chemicals likely have environmental consequences; many habitats and breeding grounds are inaccessible; and some measures are eventually to be made redundant through the growing concern of insecticide resistance. For these reasons, in addition to refining or augmenting the product pipeline for currently considered approaches - antimalarial drugs, vaccines, insecticides or otherwise - novel, complementary avenues must further be explored.

In the search for innovation, much research has shifted towards understanding the inherent biology of the mosquito itself, and the genetics of what makes it such a prolific vector for disease largely a product of its ability to reproduce in tight association with humans and their habitat, coupled with a natural susceptibility to the parasite. In this respect the presence of a well annotated genome sequence, coupled with the ability to introduce transgenes of interest, or to disrupt endogenous gene expression, help greatly (Catteruccia et al. 2000; Blandin et al. 2002; Gantz et al. 2015; Hammond et al. 2016). Not only does knowledge of the genetics of these mosquito traits help in predicting the dynamics of disease transmission, it also opens up the prospect of genetic control - that is, the deliberate introduction of genetic traits into a population for its control.

Genetic control involves the introduction of new genetic traits to a residual population through the release of insects containing the altered genetic trait that are competent to mate with extant populations. The genetic traits may be conferred by transgenes, deliberately introduced into the genome, or they may equally be non-transgenic in origin. Since it relies on mating, this type of control is species-specific, non-toxic, and has the advantage that the released insects can do the difficult task of reaching remote and otherwise infeasible insect niches.

Two main approaches of genetic control to reduce vectorial capacity are currently being explored: population suppression and population modification. In both, the numbers of permissive vectors in the target population are reduced, either in absolute terms (suppression), or by modifying potential vectors such that they cannot spread disease.

One type of genetic control that has been widely used is the sterile insect technique (SIT), which conventionally involves the release of large numbers of males that have been irradiated to produce aneuploid sperm. These males can mate with wild-type females and are able to fertilise their eggs, but the resultant embryos are inviable (Klassen and Curtis 2005). This type of technique has been used with great success in the control of many agricultural pests (Orozco-Dávila et al. 2017; Mastrangelo and Welch 2012; Zacharopoulou et al. 2017), but because it requires huge numbers of insects to be reared (in excess of the wild population) and released repeatedly for an extended period of time it is both logistically challenging and expensive. For this reason, SIT, or transgenic versions thereof such as RIDL (Release of Insects carrying a Dominant Lethal) (Thomas et al. 2000) show most utility for isolated or urban populations of vectors, where the required release ratios are more feasible to achieve. Examples of their successful application can be found in the control of Aedes aegypti populations in response to Dengue outbreaks in Brazil (Carvalho et al. 2015) or the eradication of the tsetse fly, vector of sleeping sickness, from the island of Unguja, Zanzibar (Verysen et al. 2014). 
By their very nature, genetic control strategies such as SIT that confer sterility are self-limiting, as the large reduction in fitness conferred by the trait results in its rapid removal from the population due to selection. A similar scenario applies, albeit to a lesser extent, for any population modification approach - the transgenic trait conferring refractoriness to the parasite will at best be neutral in terms of fitness conferred to the mosquito, since there is minimal fitness benefit to the mosquito from being free of parasites, and any such benefit is likely to be offset by the cost of expressing the refractoriness trait (Fig 1A) (Tripet, Aboagye-Antwi, and Hurd 2008). Thus, while these types of self-limiting strategy may hold some circumstantial advantages regarding spatiotemporal confinement (i.e. genetically modified populations are contained locally and transiently), the large-scale deployment of repeated inundative releases required to achieve area-wide control is likely to be unfeasible. Releasing at a low frequency, the trait would likely be lost over time, either due to selection or stochastic forces such as genetic drift.
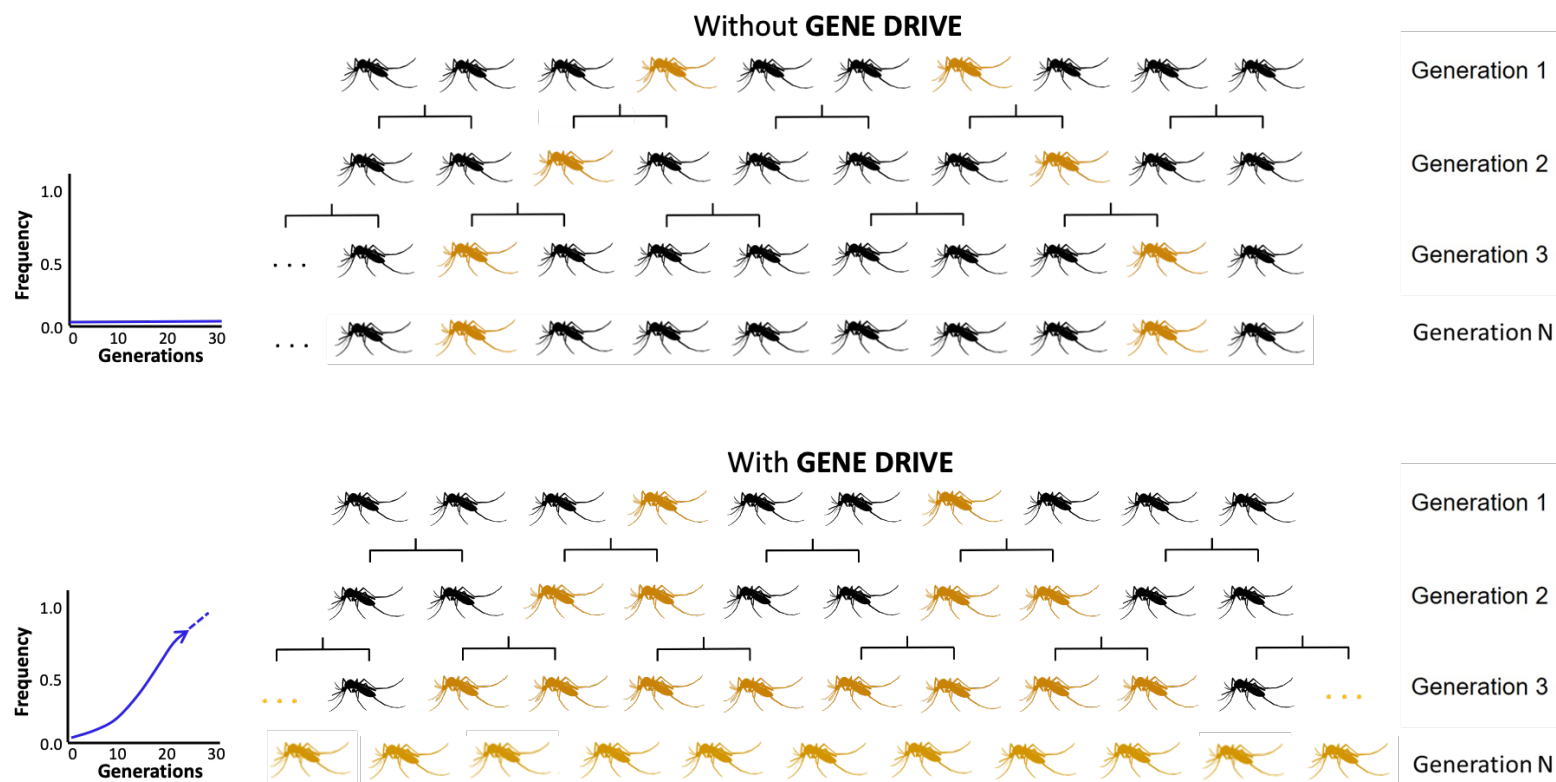

Fig. 1 Mendelian inheritance (top) versus gene drive inheritance (bottom). In sexually reproducing organisms such as mosquitoes, introducing a transgenic element through the release of modified mosquitoes (highlighted in yellow and assumed to be heterozygous here; wild type in black), transgenic individuals will transmit the transgene to $50 \%$ of their offspring. In a large population, its allelic frequency will therefore at best remain constant (top left), but more likely be lost over time due to fitness effects associated with transgene as well as the effect of genetic drift. With a gene drive, the transgenic element is preferentially inherited, such that each heterozygous individual transmits it to over $50 \%$ of its offspring each generation. In this way, the element is able to propagate throughout the population, becoming more and more frequent with each subsequent generation (bottom left), even if there is a fitness cost associated with the drive (provided the fitness cost does not outweigh the degree of biased inheritance). As the gene drive reaches a high frequency many of the individuals will be homozygous for the gene drive. For population-wide gene knockout strategies these homozygous individuals have both copies of an essential gene disrupted, meaning they are inviable in one or both sexes (depending on the target gene) and an effect on the population starts to appear.

\section{Gene drive}

To circumvent the problem of getting desirable genetic traits into a population, much contemporary research has focussed on overcoming traditional laws of Mendelian inheritance such that certain genetic elements are preferentially transmitted at rates of over $50 \%$ in the progeny, and hence become more frequent with each generation. In this way, a trait can rapidly propagate or 'drive' throughout a population despite conferring a negative effect on overall fitness, provided the distortive effect on transmission is greater than the reduction in survivability and reproduction (Fig 1B). This 
constitutes a gene drive: skewed mechanisms of genetic transmission wherein traditional Mendelian laws are superseded in order to bias the inheritance of intrinsic, 'selfish' genetic elements. 'Gene drive' is also increasingly being used to refer to the actual synthetic genetic element itself - the 'driving' component of the technology per se. Because of this biased transmission, gene drives are inoculative and self-sustaining, requiring low numbers in the initial release in order to spread and persist within a population. Thus gene drives, as opposed to other methods of genetic control, are comparatively less expensive, longer lasting and can be implemented on a much grander scale regardless of the economy and accessibility of the target community.

\section{Various flavours of drive}

There are many examples of naturally occurring gene drives and these have often been the inspiration in designing novel, engineered gene drive systems for the purposes of insect control. For example the MEDEA element, first identified in the flour beetle (Tribolium castaneum), has since been synthetically engineered in Drosophila melanogaster and comprises a toxin:antidote whereby embryos inheriting the MEDEA element (and any genetic cargo with it) survive over those that do not (Chen et al. 2007). However, success in transferring MEDEA type gene drives to other species has been limited, with the exception of the crop pest Drosophila suzukii, due to the difficulty in finding suitable toxin:antidote systems (Buchman et al. 2018). A second naturally occurring gene drive exploits genetic underdominance - a phenomenon wherein reduced fitness is attributed to the heterozygous rather than homozygous allelic state, with the latter consequently being highly selected for. In order to convert a population a relatively high frequency of individuals (in the simplest case, around a 1:1 ratio with the local wild type population) homozygous for the desirable allele needs to be released in order to fixate. Underdominance-based drives have been engineered in D. melanogaster (Akbari et al. 2013; Reeves et al. 2014), although, like MEDEA, complex means of production have hindered progress.

In contrast to some of the above approaches, nuclease-based gene drives have probably progressed further in recent years due to their relative simplicity and the advances in the site-specific nuclease technologies themselves, in part driven by the clamour for precise genome editing tools.

\section{Homing-based Gene Drives}

One class of drives take inspiration from homing endonuclease genes (HEGs), a class of naturally occurring genes which encode for various cleavage-inducing proteins and are able to persist in a population through 'cut-and-copy' repair that leads to copying of the element from one chromosome to another in a process called homing. Since the process of copying is entirely dependent on the host cell DNA repair mechanism, in principle any sequence-specific endonuclease could be repurposed to mimic a HEG (Burt 2003). Indeed, much success has been achieved recently in repurposing the sequence-specific nuclease components of the Clustered Regularly Interspaced Short Palindromic Repeats (CRISPR) system (Jinek et al. 2012; Mojica et al. 2005), namely the CRISPR-associated protein 9 (Cas9) endonuclease and the short gRNA transcription unit that provides the sequence specificity through base pairing to a target DNA sequence (Gantz and Bier 2015; Gantz et al. 2015; Hammond et al. 2016; Esvelt et al. 2014). In synthetic 'HEG-type' gene drives, the sequence encoding the endonuclease and any transgene-associated regions is inserted within a specific chromosomal locus to artificially induce heterozygosity (Fig. 2). The endonuclease recognises and cuts a specific target sequence on its homologous chromosome in order to induce a double-stranded break; the cut chromosome is in turn repaired using the intact transgene-containing chromosome as a template through the process of homology directed repair (HDR). As the nuclease gene resides within its own recognition sequence, no further cleavage takes place on the chromosome. The repair process allows the nuclease to be copied onto the other chromosome ('homing') and in doing so converts the cell 
such that both homologous chromosomes contain the gene drive. If this reaction occurs in the germline, before gamete formation, it ensures that a disproportionately high portion of the gametes (>99\%) will contain the gene drive and its associated allele.

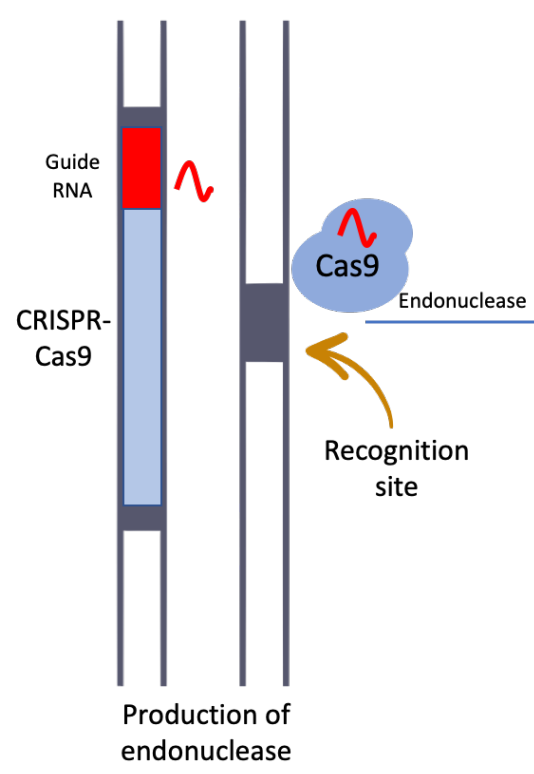

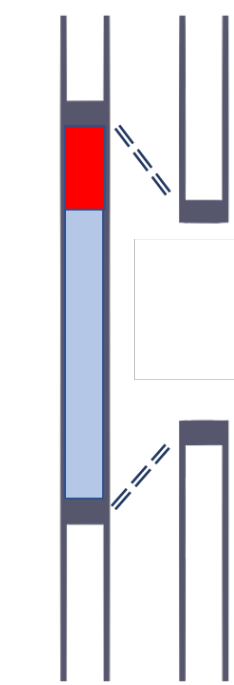

Cutting at recognition site leads to break in

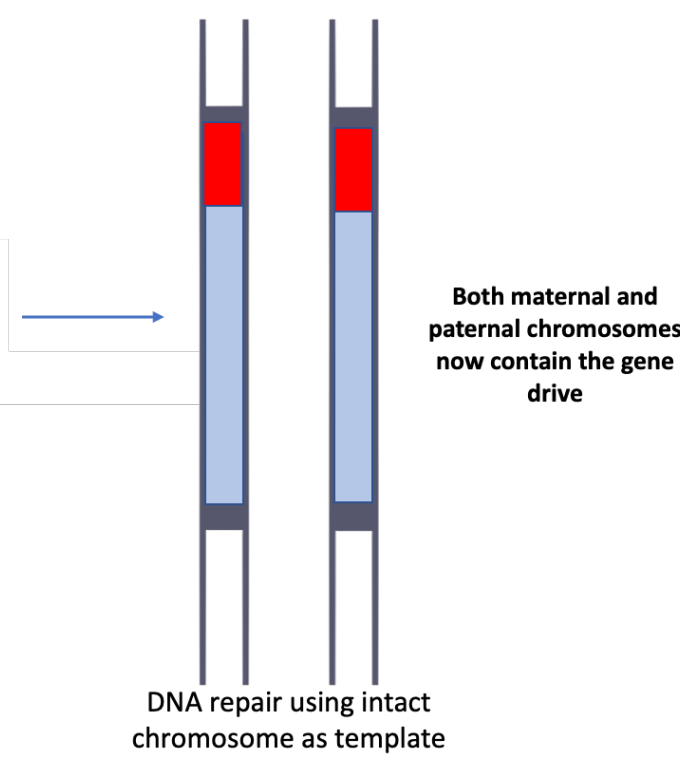

osomes drive

Fig. 2. Mechanism of nuclease-based "homing" gene drives. The endonuclease (typically CRISPR-Cas9) coding sequence is inserted within a specific genomic locus where, upon expression, it induces a double stranded break at a gRNA-targeted recognition site on the homologous wild-type chromosome. In the germline, before meiosis and partitioning of homologous chromosomes among the gametes, homology directed repair (HDR) of the cleaved chromosome using regions of homology flanking the gene drive element, leads to a copying of the transgene onto both chromosomes, using the modified chromosome as a template. This induced homozygosity ensures the gene drive element is included in nearly all the gametes, regardless of which of the two homologous chromosome they receive. In heterozygous offspring, the process repeats itself, and the gene drive is spread throughout a population over subsequent generations.

With CRISPR-based homing gene drives two approaches are distinguishable: population wide knockout of some essential gene that results in a desirable phenotype that is recessive (i.e. individuals with only one copy of the gene drive should not display the phenotype); and population wide spread of a drive with cargo in the form of an effector gene that negatively affects ability to vector the parasite (Fig 3). Proof of principle for the knockout approach was first demonstrated by a gene drive targeting a haplosufficient female fertility gene in Anopheles gambiae that could initially spread in a population despite deliberately imposing a large reproductive cost (Hammond et al. 2016). Proof of principle for the spreading of an anti-malarial effector, in the form of genes encoding for single chain antibodies that impair the parasite, has been shown with a CRISPR-based gene drive in Anopheles stephensi (Gantz et al. 2015; Pham et al. 2019). As with any population control technology, resistance mechanisms must be considered. For population-wide knockout approaches the selection pressure for such mechanisms is likely to be high in the mosquito, whereas for gene drives carrying an effector cargo this selection pressure to evolve resistance might fall more on the parasite (Marshall et al. 2019). 
Disrupt an essential gene

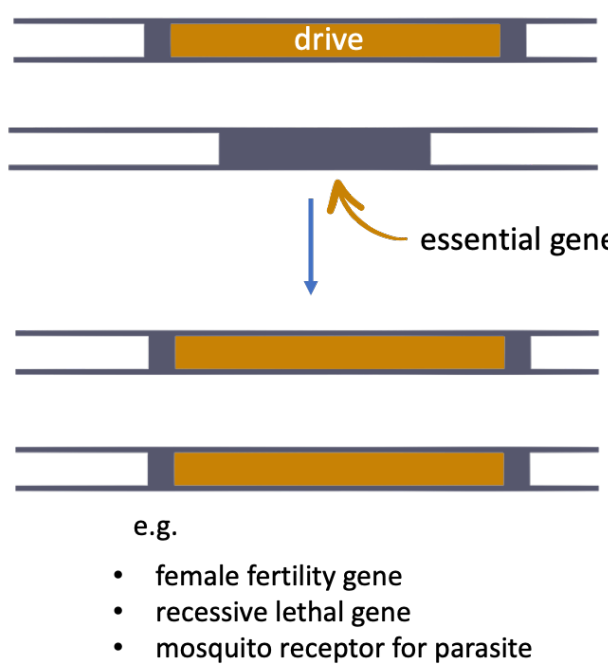

Carry an effector gene as 'cargo'

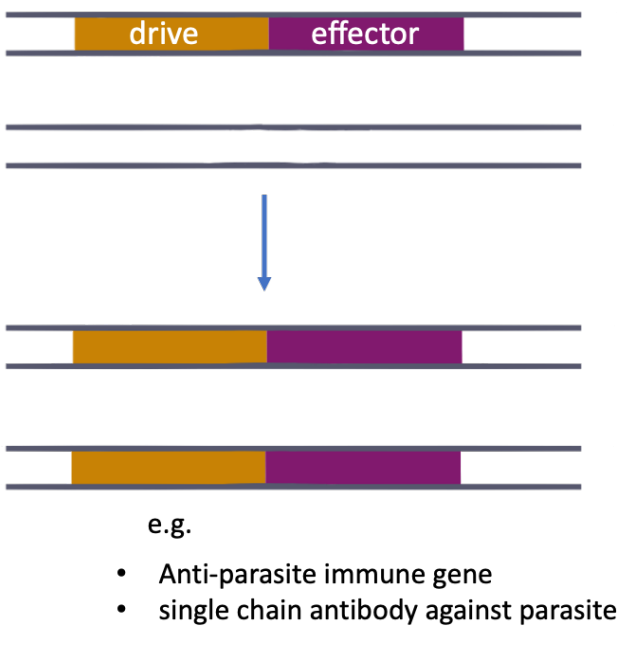

Fig. 3. Two simple types of homing gene drives. (left) Population-wide knockout of some essential gene that produces some desirable phenotype in individuals homozygous for the gene drive, such as female infertility, by virtue of the gene drive disrupting the coding sequence of the target gene. Importantly for this approach to work most effectively, 'carrier' individuals heterozygous for the drive should be fully viable. (right) Population-wide spread of an effector gene as 'cargo'. Note that mutations may occur during the homing reaction to render the effector gene non-functional, thus the cargo is effectively lost. Careful effector gene appraisal and selection can reduce the likelihood of cargo dissociation (Beaghton et al. 2017; Burt et al. 2018; Marshall et al. 2019), An alternative strategy is an 'integral' gene drive, whose components achieve the optimal expression necessary for drive by silently 'hijacking' endogenous genes without disrupting their function, a feature that ensures that each is more robust to resistance (Nash et al. 2019)

The likeliest sources of resistance in the mosquito to CRISPR-based homing drives are variant alleles in the target site recognised by the nuclease that not only prevent the nuclease cutting but confer improved fitness on the mosquito, compared to the gene drive allele. Such variant alleles may be pre-existing in the population or may be generated by the nuclease activity of the gene drive itself - cut chromosomes repaired by an alternative DNA repair, non-homologous end joining, can lead to the insertion of small insertions and deletions at the target site (Champer et al. 2017; Hammond et al. 2017). Prioritisation of target sites that show high levels of functional constraint, and thus less likely to generate tolerable mutations, is therefore a key strategy, in addition to the targeting of multiple sites in the same gene. The former approach was validated by the recent demonstration that a gene drive targeting a highly conserved region of an essential female-specific gene was able to spread rapidly to fixation in a laboratory population, to its detriment, resulting in its elimination, and without the apparent selection of any resistance (Kyrou et al. 2018). The latter approach was validated by the successful use of multiple guide RNAs within the same gene drive cassette (Kandul et al. 2020; Champer et al. 2018; Oberhofer, Ivy, and Hay 2019).

\section{Other nuclease-based drives}

Another type of nuclease-based gene drive is inspired by the observation in nature, including in Aedes and Culex genera of mosquitoes, of sex chromosomes that bias their own inheritance over the other sex chromosome, resulting in a sex bias. Since the reproductive capacity of many insect species is by and large determined by the number of females in the population, recapitulation of this phenomenon to ensure a sex ratio distortion towards males has potential for population control and was first proposed by Hamilton in the 1960s (Hamilton 1967). This has stimulated much research into the nature of sex chromosomes and the biology of sex determination, including the identification of the male-determining genes in Aedes and Anopheles mosquitoes (Hall et al. 2015; Krzywinska et al. 2016) 
with a view to manipulating their expression to ensure sex distortion. However in An. gambiae, serendipity provides that the female sex chromosomes exclusively contain several hundred copies of a highly conserved, functionally constrained target site that can be targeted by a nuclease, resulting in selective destruction of this chromosome (Galizi et al. 2014; Galizi et al. 2016; Windbichler, Papathanos, and Crisanti 2008). Expression of the nuclease during spermatogenesis results in a high sex ratio distortion (>95\% male) since sperm bearing the female sex chromosome are non-viable. Importantly though, to make this a gene drive one must ensure biased inheritance of the sex distortion construct. Conceptually, the simplest way to do this is to place the sex distorting construct on the $Y$ chromosome that is selectively inherited; however, ensuring the correct temporal and spatial expression from this largely heterochromatic chromosome is challenging. The overall approach - of targeting repeat sequences on sex-linked chromosomes to ensure sex distortion via ablation of one sex chromosome - may be broadly applicable across species other than Anopheles gambiae since a similar approach using CRISPR-Cas9 has been shown to work in Drosophila (Fasulo et al. 2019).

Some of the toxin:antidote systems of drive have similarly been enabled by the flexibility of nucleases like CRISPR-Cas9. One example is 'Cleave-and-Rescue' that comprises a single construct containing a CRISPR-Cas9 'Cleaver' component designed to act in trans to cut and inactivate an essential gene, and a 'Rescue' component linking a recoded, cleavage-resistant version of the essential gene to a transgene of interest (Oberhofer, Ivy, and Hay 2019). Offspring that do not inherit a functional copy of the essential gene thus die unless they also inherit a copy of the cleave-and-rescue construct. This provides a selective advantage to chromosomes bearing the construct (and its linked transgene) over those that do not. Since the selective disadvantage suffered by the wild type chromosome is proportional to the probability of encountering a cleave-and-rescue construct it means that the spread rate of this type of construct is frequency-dependent: very low if released at low frequencies but fast if released at high frequencies.

\section{Conclusion}

Gene drives have great potential for the control of populations and are increasingly being proposed as a cost-effective means of entomological and ecological control with vast epidemiological benefits. The applications of gene drives are not just limited to mosquito control; they have additionally been proposed as a means to control out-of-hand invasive species and to re-sensitise resistant organisms both with significant agricultural implications (Esvelt et al. 2014).

As with any intervention designed to suppress a population of insects or pathogens, selection for resistance will need to be a foremost concern in designing these gene drive strategies - the same has been true for insecticides, antibiotics and chemotherapeutics. Despite their great potential, gene drives are no exception to this rule and so robust testing during development and monitoring will be essential. Moreover, in the specific case of malaria control it is likely that no one specific technology will be a silver bullet. Rather, a combination of approaches will be essential to achieve effective and long-lasting control.

Gradually, as many technical barriers are overcome, the focus is shifting from what makes a successful gene drive to rather how and when one should be implemented. Such debates need to consider the ethical, legal and political concerns that come with using a novel technology that is designed to be invasive, and hence face major contention. In many ways, answering these questions presents as much of a challenge as overcoming the technical barriers faced by gene drives and their implementation; but a decision on whether to put the technology into practice must consider not only the risks and benefits, weighed against possible alternatives if there are any, but also, ultimately, what is the cost of doing nothing? 


\section{REFERENCES}

\section{$\bullet$ Of outstanding interest}

\section{- Of special interest}

Akbari, O. S., K. D. Matzen, J. M. Marshall, H. Huang, C. M. Ward, and B. A. Hay. 2013. 'A synthetic gene drive system for local, reversible modification and suppression of insect populations', Curr Biol, 23: 671-7.

Beaghton, A., A. Hammond, T. Nolan, A. Crisanti, H. C. Godfray, and A. Burt. 2017. 'Requirements for Driving Antipathogen Effector Genes into Populations of Disease Vectors by Homing', Genetics, 205: 1587-96.

Bhatt, S., D. J. Weiss, E. Cameron, D. Bisanzio, B. Mappin, U. Dalrymple, K. Battle, C. L. Moyes, A. Henry, P. A. Eckhoff, E. A. Wenger, O. Briet, M. A. Penny, T. A. Smith, A. Bennett, J. Yukich, T. P. Eisele, J. T. Griffin, C. A. Fergus, M. Lynch, F. Lindgren, J. M. Cohen, C. L. J. Murray, D. L. Smith, S. I. Hay, R. E. Cibulskis, and P. W. Gething. 2015. 'The effect of malaria control on Plasmodium falciparum in Africa between 2000 and 2015', Nature, 526: 207-11.

Blandin, Stéphanie, Luis F Moita, Thomas Köcher, Matthias Wilm, Fotis C Kafatos, and Elena A Levashina. 2002. 'Reverse genetics in the mosquito Anopheles gambiae: targeted disruption of the Defensin gene', EMBO reports, 3: 852-56.

Buchman, A., J. M. Marshall, D. Ostrovski, T. Yang, and O. S. Akbari. 2018. 'Synthetically engineered Medea gene drive system in the worldwide crop pest Drosophila suzukii', Proc Natl Acad Sci U $S A, 115:$ 4725-30.

Burt, A. 2003. 'Site-specific selfish genes as tools for the control and genetic engineering of natural populations', Proc Biol Sci, 270: 921-8. •• This theoretical paper describes for the first time the idea of repurposing site-specific nucleases as gene drives for population control and modification and lists a comprehensive series of considerations for their design

Burt, Austin, Mamadou Coulibaly, Andrea Crisanti, Abdoulaye Diabate, and Jonathan K. Kayondo. 2018. 'Gene drive to reduce malaria transmission in sub-Saharan Africa', Journal of Responsible Innovation, 5: S66-S80.

Carvalho, D. O., A. R. McKemey, L. Garziera, R. Lacroix, C. A. Donnelly, L. Alphey, A. Malavasi, and M. L. Capurro. 2015. 'Suppression of a Field Population of Aedes aegypti in Brazil by Sustained Release of Transgenic Male Mosquitoes', PLoS Negl Trop Dis, 9: e0003864.

Catteruccia, Flaminia, Tony Nolan, Thanasis G. Loukeris, Claudia Blass, Charalambos Savakis, Fotis C. Kafatos, and Andrea Crisanti. 2000. 'Stable germline transformation of the malaria mosquito Anopheles stephensi', Nature, 405: 959-62.

Champer, J., R. Reeves, S. Y. Oh, C. Liu, J. Liu, A. G. Clark, and P. W. Messer. 2017. 'Novel CRISPR/Cas9 gene drive constructs reveal insights into mechanisms of resistance allele formation and drive efficiency in genetically diverse populations', PLoS Genet, 13: e1006796.

Champer, Jackson, Jingxian Liu, Suh Yeon Oh, Riona Reeves, Anisha Luthra, Nathan Oakes, Andrew G. Clark, and Philipp W. Messer. 2018. 'Reducing resistance allele formation in CRISPR gene drive', Proceedings of the National Academy of Sciences, 115: 5522-27. •

Chen, Chun-Hong, Haixia Huang, Catherine M. Ward, Jessica T. Su, Lorian V. Schaeffer, Ming Guo, and Bruce A. Hay. 2007. 'A Synthetic Maternal-Effect Selfish Genetic Element Drives Population Replacement in Drosophila', Science, 316: 597-600.

Esvelt, Kevin M., Andrea L. Smidler, Flaminia Catteruccia, and George M. Church. 2014. 'Concerning RNA-guided gene drives for the alteration of wild populations', Elife, 3: e03401. •• This theoretical paper takes the ideas of Burt and adapts the design considerations to the CRISPR-Cas9 family of nucleases

Fasulo, Barbara, Angela Meccariello, Maya Morgan, Carl Borufka, Philippos Aris Papathanos, and Nikolai Windbichler. 2019. 'A fly model establishes distinct mechanisms for synthetic CRISPR/Cas9 sex distorters', bioRxiv: 834630. 
Galizi, R., L. A. Doyle, M. Menichelli, F. Bernardini, A. Deredec, A. Burt, B. L. Stoddard, N. Windbichler, and A. Crisanti. 2014. 'A synthetic sex ratio distortion system for the control of the human malaria mosquito', Nat Commun, 5: 3977. •

Galizi, R., A. Hammond, K. Kyrou, C. Taxiarchi, F. Bernardini, S. M. O'Loughlin, P. A. Papathanos, T. Nolan, N. Windbichler, and A. Crisanti. 2016. 'A CRISPR-Cas9 sex-ratio distortion system for genetic control', Sci Rep, 6: 31139.

Gantz, V. M., N. Jasinskiene, O. Tatarenkova, A. Fazekas, V. M. Macias, E. Bier, and A. A. James. 2015. 'Highly efficient Cas9-mediated gene drive for population modification of the malaria vector mosquito Anopheles stephensi', Proc Natl Acad Sci U S A, 112: E6736-43. •• This paper describes the first practical demonstration of a gene drive designed to contain a cargo gene whose product impairs transmission of the malaria parasite through mosquitoes

Gantz, Valentino M., and Ethan Bier. 2015. 'The mutagenic chain reaction: A method for converting heterozygous to homozygous mutations', Science, 348: 442-44. •

Hall, Andrew Brantley, Sanjay Basu, Xiaofang Jiang, Yumin Qi, Vladimir A. Timoshevskiy, James K. Biedler, Maria V. Sharakhova, Rubayet Elahi, Michelle A. E. Anderson, Xiao-Guang Chen, Igor V. Sharakhov, Zach N. Adelman, and Zhijian Tu. 2015. 'A male-determining factor in the mosquito <em>Aedes aegypti</em>', Science, 348: 1268-70.

Hamilton, W. D. 1967. 'Extraordinary Sex Ratios', Science, 156: 477-88.

Hammond, A., R. Galizi, K. Kyrou, A. Simoni, C. Siniscalchi, D. Katsanos, M. Gribble, D. Baker, E. Marois, S. Russell, A. Burt, N. Windbichler, A. Crisanti, and T. Nolan. 2016. 'A CRISPR-Cas9 gene drive system targeting female reproduction in the malaria mosquito vector Anopheles gambiae', Nat Biotechnol, 34: 78-83. • Describes the first gene drive aimed at population suppression, designed to spread in a population of mosquitoes while reducing the reproductive output of females.

Hammond, A. M., K. Kyrou, M. Bruttini, A. North, R. Galizi, X. Karlsson, N. Kranjc, F. M. Carpi, R. D'Aurizio, A. Crisanti, and T. Nolan. 2017. 'The creation and selection of mutations resistant to a gene drive over multiple generations in the malaria mosquito', PLoS Genet, 13: e1007039. •

Jinek, Martin, Krzysztof Chylinski, Ines Fonfara, Michael Hauer, Jennifer A. Doudna, and Emmanuelle Charpentier. 2012. 'A Programmable Dual-RNA-Guided DNA Endonuclease in Adaptive Bacterial Immunity', Science, 337: 816-21.

Kandul, Nikolay P., Junru Liu, Anna Buchman, Valentino M. Gantz, Ethan Bier, and Omar S. Akbari. 2020. 'Assessment of a Split Homing Based Gene Drive for Efficient Knockout of Multiple Genes', G3: Genes/Genomes/Genetics, 10: 827-37.

Klassen, W., and C. F. Curtis. 2005. History of the sterile insect technique (Springer: Netherlands).

Krzywinska, Elzbieta, Nathan J. Dennison, Gareth J. Lycett, and Jaroslaw Krzywinski. 2016. 'A maleness gene in the malaria mosquito <em>Anopheles gambiae</em>', Science, 353: 67-69.

Kyrou, K., A. M. Hammond, R. Galizi, N. Kranjc, A. Burt, A. K. Beaghton, T. Nolan, and A. Crisanti. 2018. 'A CRISPR-Cas9 gene drive targeting doublesex causes complete population suppression in caged Anopheles gambiae mosquitoes', Nat Biotechnol, 36: 1062-66. •• The first demonstration of population crash using a gene drive re-designed to be more robust and less susceptible to the development of resistance.

Marshall, John M., Robyn R. Raban, Nikolay P. Kandul, Jyotheeswara R. Edula, Tomás M. León, and Omar S. Akbari. 2019. 'Winning the Tug-of-War Between Effector Gene Design and Pathogen Evolution in Vector Population Replacement Strategies', Frontiers in Genetics, 10.

Mastrangelo, T., and J. B. Welch. 2012. 'An Overview of the Components of AW-IPM Campaigns against the New World Screwworm', Insects, 3: 930-55.

Mojica, Francisco J. M., Chc sar Díez-Villaseñor, Jesús García-Martínez, and Elena Soria. 2005. 'Intervening Sequences of Regularly Spaced Prokaryotic Repeats Derive from Foreign Genetic Elements', Journal of Molecular Evolution, 60: 174-82. 
Nash, Alexander, Giulia Mignini Urdaneta, Andrea K. Beaghton, Astrid Hoermann, Philippos Aris Papathanos, George K. Christophides, and Nikolai Windbichler. 2019. 'Integral gene drives for population replacement', Biology Open, 8: bio037762.

Oberhofer, G., T. Ivy, and B. A. Hay. 2019. 'Cleave and Rescue, a novel selfish genetic element and general strategy for gene drive', Proc Natl Acad Sci U S A, 116: 6250-59.

Orozco-Dávila, Dina, Luis Quintero, Emilio Hernández, Eduardo Solís, Trinidad Artiaga, Refugio Hernández, Carlos Ortega, and Pablo Montoya. 2017. 'Mass rearing and sterile insect releases for the control of Anastrepha spp. pests in Mexico - a review', Entomologia Experimentalis et Applicata, 164: 176-87.

Pham, T. B., C. H. Phong, J. B. Bennett, K. Hwang, N. Jasinskiene, K. Parker, D. Stillinger, J. M. Marshall, R. Carballar-Lejarazu, and A. A. James. 2019. 'Experimental population modification of the malaria vector mosquito, Anopheles stephensi', PLoS Genet, 15: e1008440.

Reeves, R. G., J. Bryk, P. M. Altrock, J. A. Denton, and F. A. Reed. 2014. 'First steps towards underdominant genetic transformation of insect populations', PLoS One, 9: e97557.

Thomas, D. D., C. A. Donnelly, R. J. Wood, and L. S. Alphey. 2000. 'Insect Population Control Using a Dominant, Repressible, Lethal Genetic System', Science, 287.

Tripet, Frédéric, Fred Aboagye-Antwi, and Hilary Hurd. 2008. 'Ecological immunology of mosquitomalaria interactions', Trends in Parasitology, 24: 219-27.

Verysen, M. J. B., K. Saleh, F. Mramba, A. Parker, U. Feldmann, V. A. Dyck, A. Msangi, and J. Bouyer. 2014. 'Sterile Insects to Enhance Agricultural Development: The Case of Sustainable Tsetse Eradication on Unguja Island, Zanzibar, Using an Area-Wide Integrated Pest Management Approach', PLoS Negl Trop Dis, 8.

Windbichler, N., P. A. Papathanos, and A. Crisanti. 2008. 'Targeting the $X$ chromosome during spermatogenesis induces $Y$ chromosome transmission ratio distortion and early dominant embryo lethality in Anopheles gambiae', PLoS Genet, 4: e1000291.

World Health Organisation. 2017. 'Vector-borne diseases', Accessed December 2019. https://www.who.int/news-room/fact-sheets/detail/vector-borne-diseases.

--_. 2019. 'Guidelines for Malaria Vector Control', Accessed December 2019. https://www.who.int/malaria/publications/atoz/9789241550499/en/.

Zacharopoulou, A., A. A. Augustinos, E. Drosopoulou, K. T. Tsoumani, A. Gariou-Papalexiou, G. Franz, K. D. Mathiopoulos, K. Bourtzis, and P. Mavragani-Tsipidou. 2017. 'A review of more than 30 years of cytogenetic studies of Tephritidae in support of sterile insect technique and global trade', Entomologia Experimentalis et Applicata, 164: 204-25. 